\title{
Morphing Planar Graphs in Spherical Space`
}

\author{
Stephen G. Kobourov and Matthew Landis \\ Department of Computer Science \\ University of Arizona \\ \{kobourov, mlandis\}@cs.arizona.edu
}

\begin{abstract}
We consider the problem of intersection-free planar graph morphing, and in particular, a generalization from Euclidean space to spherical space. We show that there exists a continuous and intersectionfree morph between two sphere drawings of a maximally planar graph, provided that both sphere drawings have convex inscribed polytopes, where sphere drawings are the spherical equivalent of plane drawings: intersection-free geodesic-arc drawings. In addition, we describe a morphing algorithm along with its implementation. Movies of sample morphs can be found at http://www.cs.arizona.edu/ mlandis/smorph.
\end{abstract}

\section{Introduction}

Morphing refers to the process of transforming one shape (the source) into another (the target). Morphing is widely used in computer graphics, animation, and modeling; see a survey by Gomes et al. 8]. In planar graph morphing we would like to transform a given source graph to another pre-specified target graph. A smooth transformation of one graph into another can be useful when dealing with dynamic graphs and graphs that change through time where it is crucial to preserve the mental map of the user. The mental map preservation is often accomplished by minimizing the changes to the drawing and by creating smooth transitions between consecutive drawings.

In this paper we consider the problem of morphing between two drawings, $D_{s}$ and $D_{t}$, of the same maximally planar graph $G=(V, E)$ on the sphere, where maximally planar graphs (or fully-triangulated graphs) are planar graphs in which every face is a triangle. The source drawing $D_{s}$ and the target drawing $D_{t}$ are sphere drawings (generalizations of Euclidean plane drawings to spherical space). The main objective is to find a continuous and intersection-free morph from $D_{s}$ to $D_{t}$. Note that the restriction to maximally planar graphs is not a loss of generality, as planar graphs are easily augmented to maximally planar.

\subsection{Previous Work}

Morphing has been extensively studied in graphics, animation, modeling and computational geometry, e.g., morphing 2D images [10, polygons and polylines [14, 3D objects 11] and free form curves [13.

\footnotetext{
^ This work is supported in part by NSF grants CCF-0545743 and ACR-0222920.
} 
Graph morphing, refers to the process of transforming a given graph $G_{1}$ into another graph $G_{2}$. Early work on this problem includes a result by Cairns in 1944 [4 who shows that if $G_{1}$ and $G_{2}$ are maximally planar graphs with the same embedding, then there exists a non-intersecting morph between them. Later, Thomassen [16] showed that if $G_{1}$ and $G_{2}$ are isomorphic convex planar graphs with the same outer face, then there exists a non-intersecting morph between them that preserves convexity. Erten et al. show how to morph between drawings with straight-line segments, bends, and curves [ 6 . This algorithm makes use of compatible triangulations [2] and the convex representation of a graph via barycentric coordinates 7/17.

While Thomassen [16] proved that an intersection-free morph exists, his approach neither provides a polynomial bound on the number of steps needed, nor yields a practical morphing algorithm. Floater and Gotsman [7] and Gotsman and Surazhsky [1015] describe practical morphing techniques, although these approaches neither compute explicit vertex trajectories, nor guarantee a polynomial bound on the complexity of these trajectories. Recently, Lubiw et al. 12. developed the first algorithm for intersection-free morphing with well-behaved complexity for a special case of graphs drawings, namely, orthogonal graph drawings. This work follows an earlier result by Biedl et al. $[3$ where each edge has the same bends in the same direction in the source and target drawings.

As the sphere and the plane are topologically the same, it is natural to attempt to generalize the non-intersecting morph algorithm from Euclidean space to spherical space. Alfeld et al. [1] and Gotsman et al. 9] define analogues of barycentric coordinates on the sphere, for spherical Bernstein-Bézieri polynomials and for spherical mesh parameterization, respectively. However, barycentric coordinates are problematic in spherical space. One problem is that unlike on the Euclidean plane, three points on a sphere define two finite regions. A system of barycentric coordinates must distinguish between these two regions. A second problem arises from the non-linearity introduced by the sphere. The system of equations used to determine the drawing at any stage of the morph has non-unique solutions, and it is not easy to guarantee smoothness of the morph.

\subsection{Our Results}

Our approach to morphing spherical drawings focuses on affine transformations of the inscribed polytopes of the given spherical drawings. The inscribed polytope of a spherical drawing is obtained by replacing the geodesic edges by straight-line segments. We apply rotations, translations, scaling and shearing to the inscribed polytope, while projecting its endpoints onto the surface of the sphere throughout the transformations. At an intermediate stage, we use the intersection-free morphing algorithm for plane drawings together with a gnomonic projection to/from the sphere. Our approach yields a continuous and intersection-free morph for sphere drawings of maximally planar graphs, provided that the source and target drawings have convex inscribed polytopes. Note that in general, the inscribed polytope of a sphere drawing is star-shaped, though not necessarily convex. Therefore, while we do not resolve the general 
problem of morphing spherical drawings, we describe an approach which works for a subclass of spherical drawings and which hopefully can be used to resolve the general problem.

\section{Background}

We begin with some mathematical background about sphere drawings and spherical projections. The concept of a straight line in Euclidean space generalizes to that of a geodesic in Riemannian spaces, where the geodesic between two points is defined as a continuously differentiable curve of minimal length between them. Thus, geodesics in Euclidean geometry are straight lines, and in spherical geometry they are arcs of great circles. The generalization of an intersection-free straight-line drawing of a planar graph in spherical space uses geodesics instead of straight-lines.

Definition 1. A sphere embedding of a graph is a clockwise order of the neighbors for each vertex in the graph. A drawing is a drawing of an embedding if neighbors of nodes in the drawing match the order in the embedding. Note that 3 -connected planar graphs in general, and maximally graphs in particular, have a unique sphere embedding, up to reflection.

Definition 2. A geodesic-arc sphere drawing of a graph is the sphere analogue of a straight-line drawing of a graph. The drawing is determined entirely by a mapping of the vertices of the graph onto the sphere. An edge between two nodes is drawn as the geodesic arc between them. We assume that no two nodes are antipodal, as there is no unique geodesic arc between two antipodal points.

Definition 3. An intersection-free, geodesic-arc sphere drawing of a graph is a sphere drawing of the graph in which no two edges intersect, except at a node on which they are both incident. We refer to such drawings as sphere drawings for short. Note that sphere drawings are a generalization of straight-line plane drawings from Euclidean space to spherical space.

Definition 4. Given a sphere drawing $D$ of a planar graph $G$, the inscribed polytope $P$ of $D$ is obtained by replacing the (geodesic) edges in the spherical drawing by straight-line segments. The inscribed polytope $P$ is by definition simple and star-shaped, though not necessarily convex.

Definition 5. The gnomonic projection is a non-conformal map projection obtained by projecting a point on the surface of the sphere from the sphere's center to the point in a plane that is tangent to the south pole. Since this projection sends antipodal points to the same point on the plane, it can only be used to project one hemisphere at a time. In a gnomonic projection, geodesics are mapped to straight lines and vice versa [5].

Note that a stereographic projection from the sphere to the plane, with the north pole as the focus of the projection, unambiguously maps each point from 


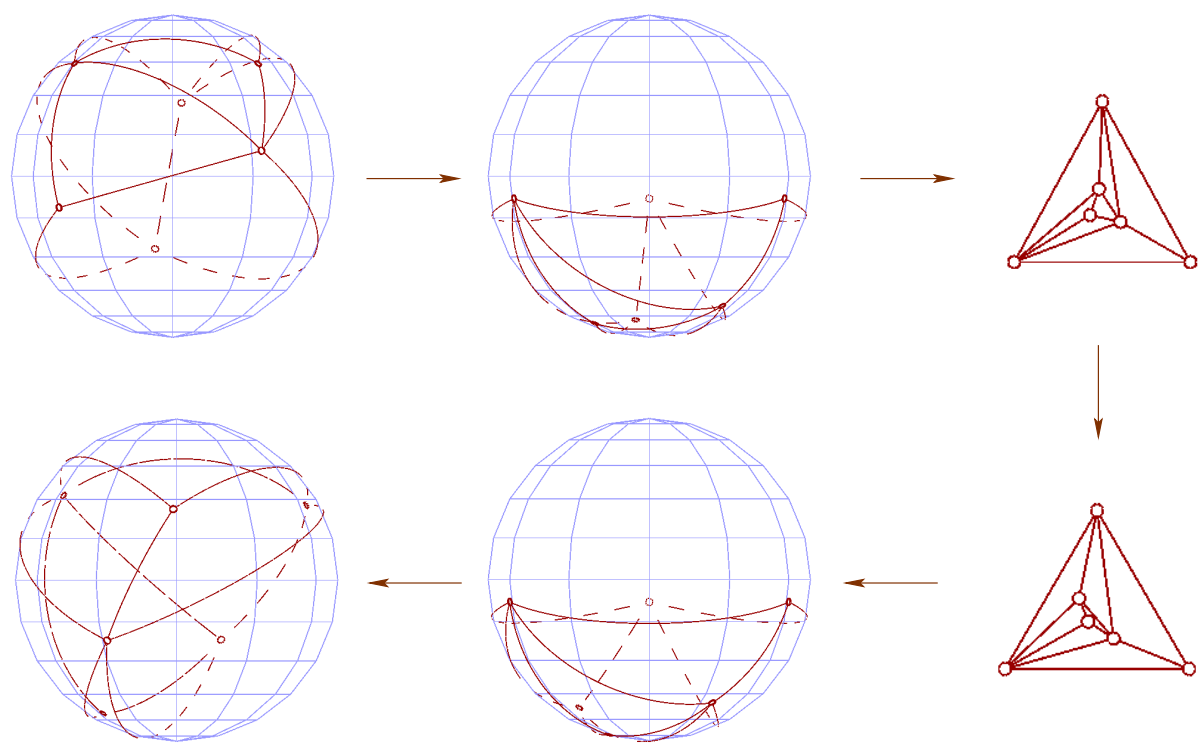

Fig. 1. Screenshots from our implementation, illustrating the morphing sequence: $D_{s} \rightarrow D_{s}^{\prime} \rightarrow D_{s}^{\prime \prime} \rightarrow D_{t}^{\prime \prime} \rightarrow D_{t}^{\prime} \rightarrow D_{t}$

the sphere to a point on the plane. In this case, however, a sphere drawing is mapped to an intersection-free drawing of the graph in the plane but that drawing is not a straight-line one. As the graph morphing algorithm for plane drawings assumes edges are straight-line segments, we use a gnomonic projection.

\section{Morphing Between Sphere Drawings}

The algorithm for morphing between two sphere drawings $D_{s}$ and $D_{t}$ of the same underlying graph $G$ can be broken into several stages:

1. Choose an outer face $f_{0}$ of the underlying graph;

2. Morph the source sphere drawing $D_{s}$ of $G$ into $D_{s}^{\prime}$, where $D_{s}^{\prime}$ is a sphere drawing of $G$ such that the north pole is inside $f_{0}$ and the entire drawing is below the equator;

3. Morph the target sphere drawing $D_{t}$ of $G$ into $D_{t}^{\prime}$, where $D_{t}^{\prime}$ is a sphere drawing of $G$ such that the north pole is inside $f_{0}$ and the entire drawing is below the equator;

4. Project $D_{s}^{\prime}$ and $D_{t}^{\prime}$ using a gnomonic projection onto the plane tangent to the south pole to the drawings $D_{s}^{\prime \prime}$ and $D_{t}^{\prime \prime}$;

5. Morph $D_{s}^{\prime \prime}$ into $D_{t}^{\prime \prime}$ using the morphing algorithm for plane drawings [6].

In practice, step 3 of the above algorithm is used in the reverse direction and altogether, the morphing sequence is: $D_{s} \rightarrow D_{s}^{\prime} \rightarrow D_{s}^{\prime \prime} \rightarrow D_{t}^{\prime \prime} \rightarrow D_{t}^{\prime} \rightarrow D_{t}$; see 

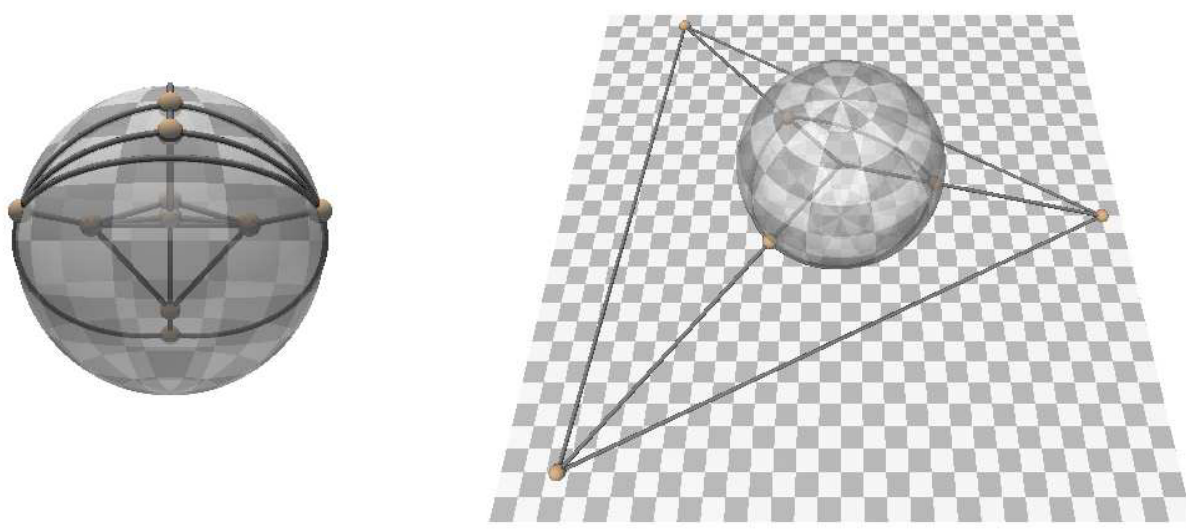

(a)

Fig. 2. (a) Projecting from a polytope that contains the origin to the surface of the sphere; (b) Gnomonic projection to and from the sphere

Fig. 1. By the definition of a gnomonic projection, since $D_{s}^{\prime}$ and $D_{t}^{\prime}$ are both strictly in the lower hemisphere, their projections $D_{s}^{\prime \prime}$ and $D_{t}^{\prime \prime}$ onto the plane tangent to the south pole are plane drawings. This implies the correctness of steps 4 and 5 and so, to argue the correctness of the overall approach, we must show that steps 2 and 3 of the algorithm above can be accomplished without introducing crossings in the morph.

\subsection{Maintaining a Smooth and Intersection-Free Morph}

Our approach to morphing sphere drawings uses a series of affine transformations to the inscribed polytope of the underlying graph (steps 2 and 3). We also rely on the barycentric morphing approach for plane drawings (steps 4 and 5). Thus, throughout the morph of our sphere drawing, we often track two positions for each vertex: the actual position of the vertex on the sphere in the sphere drawing, and the other, in some other construct, such as a 3D polytope, as in Fig. 2(a), or a plane drawing, as in Fig. 2(b). When transformations to the construct are applied, the positions of the vertices on the sphere change appropriately. A useful visualization for this approach is to imagine a spoke for each vertex, going from the origin of the sphere through both positions associated with that node. As one position changes, so does the other. For simplicity, assume the sphere is centered at $(0,0,0)$ with radius 1 and that the projection plane is $z=-1$.

Our first results deal with projecting a polytope on the surface of a sphere and the effect of affine transformations on the polytope to its projection.

Theorem 1. A strictly convex polytope containing the center of a sphere yields a sphere drawing of that polytope's skeletal graph when its vertices are normalized to lie on the sphere. 
Proof Sketch: First, note that the geodesic arc between two vertices on the sphere is the same as the projection of the straight line between those two vertices of the polytope. Suppose that the projection of the polytope onto the sphere has a crossing. Consider the point $p$ on the sphere where two edges intersect. This point must be the projection of two different polytope edges onto the sphere. This implies that there exists a ray that starts at the center and intersects two separate edges of the polytope. Let $p_{1}$ and $p_{2}$ be the two points obtained from the intersection of each of these edges with the ray through the origin. Without loss of generality, let $p_{1}$ be the point that is further from the center. Then there exists a line segment from the center of the sphere to $p_{1}$ that passes through $p_{2}$. This contradicts the assumption that the polytope is strictly convex. Hence, the resulting sphere drawing must be intersection-free.

Affine transformations of a convex polytope result in a convex polytope [5]. This observation, together with Theorem 1 yields the following Theorem:

Theorem 2. Affine transformations to a convex polytope $P$ that contains the center of a sphere, result in sphere drawings of that polytope's skeletal graph when its vertices are normalized to lie on the sphere, if the origin remains inside $P$ throughout the transformation.

As we are not assuming that the inscribed polytope obtained from a sphere drawing contains the origin, and we propose to deal with sphere drawings strictly contained in the lower hemisphere, we need an analogous theorem dealing with polytopes not containing the origin.

Theorem 3. A strictly convex polytope $P$ not containing the center of a sphere yields a sphere drawing of that polytope's skeletal graph when its vertices are normalized to lie on the sphere if, for some face $f_{1}$, the ray from the origin to any point on the polytope intersects $f_{1}$ before any other part of the polytope, and none of the faces of $P$ lie in planes containing the origin.

Proof Sketch: The face $f_{1}$ acts as a shield for rays emanating from the origin. Given a point $p$ of the polytope we can determine its projection $p^{\prime}$ on the surface of the sphere by taking the intersection of the ray from $(0,0,0)$ through $p$ with the sphere. As in Theorem 1, we may have a crossing in the spherical drawing if the ray passes through more than one edge of $P$. Since $P$ is convex, the ray intersects $P$ 's faces in at most two places. If the ray hits fewer than two faces, then clearly it is not going to intersect two edges.

Consider the cases where the ray enters $P$ through face $f_{1}$ (by assumption, it must) and exits through some other point. If the ray does not intersect an edge of $f_{1}$ at its entry point, then the only place at which it can intersect an edge of $P$ is its exit point. There can thus be at most one intersection, and we cannot have a crossing from this. If the ray hits an edge of $f_{1}$ at its entry point then, since $f_{1}$ shields all other faces from the origin, for the ray to hit another edge at its exit point, there would have to be a face adjacent to $f_{1}$ lying in a plane containing the origin (such that the ray would pass through the edge of $f_{1}$ and 


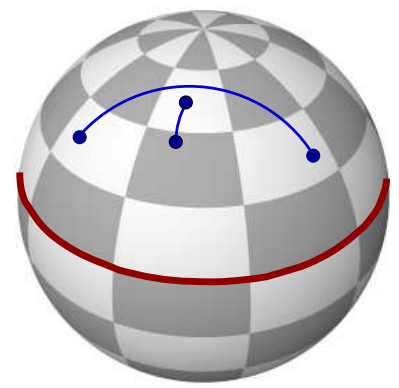

(a)

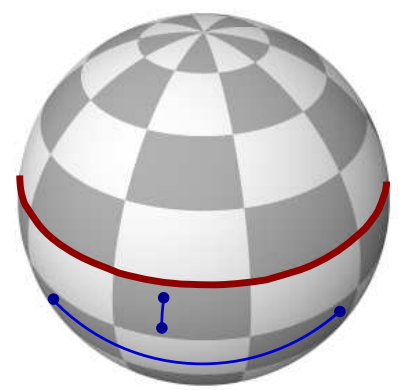

(b)

Fig. 3. Linear scaling of the vertices to the southern hemisphere may introduce crossings: (a) the endpoints of the long edge are below those of the short edge; (b) linear scaling could bring all the vertices to the southern hemisphere but at some intermediate stage the two edges intersect

remain in the adjacent face until it exits $P$ through another of that face's edges). This case is disallowed by another of the theorem's assumptions.

\subsection{Sliding Sphere Drawings to the Equator}

The obvious method of "sliding" a sphere drawing down to the lower hemisphere is to do a simple linear scale of the drawing, either by z-coordinates in Euclidean coordinates, or by $\phi$ in spherical coordinates. This approach, however, does not always work. It is easy to construct an example with two nonintersecting geodesics in the upper hemisphere that must cross on their way to the lower hemisphere if linear scaling is used; see Fig. 3. Therefore, we consider the approach where we manipulate the inscribed polytope.

Theorem 4. There exists a continuous and intersection-free morph that moves a sphere drawing $D$, of a maximally planar graph $G$, to a drawing of $G$ such that the vertices of a chosen face $f_{0}$ are on the equator and all others are strictly below the equator, provided that the inscribed polytope $P$ of $D$ is convex.

Proof Sketch: Consider the inscribed convex polytope $P$ corresponding to the sphere drawing $D$. We have two cases: either $P$ contains the origin or it does not.

Case 1 ( $P$ contains the origin): First rotate $P$ so that the outward normal to $f_{0}$ is parallel to $(0,0,1)$. Let $v_{0}$ be the average of the points of $f_{0}$. Since $P$ is convex, the segment between the origin and $v_{0}$ lies entirely within $P$. We can thus apply to $P$ a translation along the vector $-v_{0}$ and be assured that $P$ contains the origin throughout the transformation, hence Theorem 1 applies. Now $f_{0}$ lies within the $x y$-plane, so when we project its points onto the sphere, 
they lie on the equator. Since $P$ is convex, we know all other points of $P$ are on one side of $f_{0}$. Since the outward normal of $f_{0}$ is pointing up, the other points are then below $f_{0}$, and hence below the equator.

Case 2 ( $P$ does not contain the origin): Here we rely on Theorem 3 , instead. First we need to show that its preconditions are true: that there exists some face $f_{1}$ that acts as an shield that eclipses the rest of the polytope from the origin, and no faces lie in planes containing the origin.

Since $P$ does not contain the origin, there exists some plane that passes through the origin such that $P$ lies entirely on one side of that plane. Thus $D$ has one face, which we conveniently call $f_{1}$, which encompasses a half-sphere. The face $f_{1}$ must eclipse the rest of $P$ from the origin. The edges in $D$ that make up $f_{1}$ match the edge of the spherical region eclipsed by $f_{1}$ in $P$. Since $f_{1}$ is the outermost face, there can be no nodes outside of this region.

The second condition is straightforward: the only way three points on a sphere can lie on a plane containing the origin is if they all lie on a great circle (a circle whose center is the same as the sphere's). A face made by three such points is either defined by a great circle, in which case the face itself, and hence $P$, contains the origin (so we would have already dealt with it by case 1 ), or the three points all lie within a half-circle, in which case the arc between the two most distant completely overlaps the arcs between the other two pairs, in which case we did not have a valid sphere drawing to begin with.

We have shown Theorem 3 applies, and can thus apply any affine transformations to $P$ that maintains $f_{1}$ 's eclipse of the rest of the polytope. We use shearing, as it is an affine transformation and straight lines remain straight. If the application of a transformation were to negate $f_{1}$ 's "eclipse" property, then it would have to introduce a clear path from the origin to some edge in $P$ not on $f_{1}$.

Shearing and rotation do not affect the origin, so we can apply these transformations (around the origin, anyway) while maintaining a valid sphere drawing in the projection. Let $v_{0}$ be the centroid of $f_{1}$. We rotate $P$ so that $v_{0}$ lies in the $x y$-plane on the line $y=x$. Now $v_{0}$ lies at $(a, a, 0)$, for some $a$. Simultaneously shear $P$ in $x$ and $y$ with the factor -1 , so that $v_{0}$ ends up at the origin. We now have a convex polyhedron that contains the origin, and we have reduced the problem to case 1 .

\subsection{Sliding Sphere Drawings to the Lower Hemisphere}

From Theorem 4 we know that we can transform $D$ into a drawing such that the vertices of a face $f_{0}$ are on the equator and all the rest are strictly below the equator. At this stage it is easy to argue that there exists an $\epsilon>0$ such that we can translate the polytope by an additional $\epsilon$ vertically down, so that all the points on the sphere (including those that form $f_{0}$ ) are strictly below the equator.

In practice, however, the valid values of $\epsilon$ can be arbitrarily small, making this simple approach unattractive for morphing. The value of $\epsilon$ depends on the 
placement of the vertices of $f_{0}$ around the equator. If two vertices of $f_{0}$ are nearantipodal, then the edge between them can pass arbitrarily close to the south pole when we translate $P$ strictly below the equator. This would make it difficult to prevent crossings in the spherical drawing. Instead, we use scaling and shearing (both affine transformations) of the polytope $P$ to make $f_{0}$ an equilateral triangle. We consider $f_{0}$ by itself in the plane, calculate the transformations necessary to make it equilateral (shear around its centroid until it is isosceles, and then scale to make it equilateral), and apply them to $P$ as a whole.

Our goal is to move all vertices outside of $f_{0}$ low enough on the sphere so that we can guarantee $f_{0}$ blocks their view of the origin. As we show below, it suffices to move the rest of the points below the Antarctic circle $\left(66^{\circ} \mathrm{S}, z \approx-0.9135\right)$ to ensure that they are eclipsed by an $f_{0}$ whose vertices lie on the Tropic of Capricorn $\left(23.5^{\circ} \mathrm{S}, z \approx-0.3987\right)$. These two values also provide a bound on the area of the straight-line plane drawing obtained as the gnomonic projection of the sphere drawing. With the next theorem we derive the general relation that must exist between these two latitudes in order to guarantee we obtain an intersection-free sphere drawing, as per Theorem 3, and it is straight-forward to verify that that these two values satisfy the relation.

Theorem 5. There exists a continuous and intersection-free morph that moves a sphere drawing $D$, of a maximally planar graph $G$, to a drawing of $G$ such that all the vertices are strictly below the equator, provided that the inscribed polytope $P$ of $D$ is convex.

Proof Sketch: Here is the outline of the proof. We begin with $f_{0}$ as a triangle in the $x y$-plane. We apply scaling and shearing to $P$ to transform $f_{0}$ into an equilateral triangle. We choose a value $z_{1}$ that we want to translate $f_{0}$ down to, and calculate a scaling factor $s$ as a function of $z_{1}$ and $z_{3}$, the highest $z$ coordinate of any point outside $f_{0}$. We scale $P$ in $x$ and $y$ by a factor of $\frac{1}{s}$, and project it back onto the sphere. Note that this leaves $f_{0}$ in the $x y$-plane. The scaling factor was computed so that when we translate $P$ down by $z_{1}$ the face $f_{0}$ eclipses the rest of $P$, yielding a valid sphere drawing at each stage by Theorem 3. Since $f_{0}$ is now strictly below the equator, and all other nodes are below $f_{0}$, the entire drawing is below the equator. Next we provide some of the details about this argument.

We begin where Theorem 4 left off. The inscribed polytope $P$ has the designated face $f_{0}$ on the equator and all other vertices in the southern hemisphere. We skip the details about scaling and shearing to $P$ to transform $f_{0}$ into an equilateral triangle, and focus on calculating the scaling factor $s$ needed to ensure that when we translate $P$ below the equator, the spherical drawing contains no crossings.

Since we have transformed $f_{0}$ into an equilateral triangle, we know exactly where its arcs lie, and can calculate the lowest point on the sphere covered by $f_{0}$. We would like to translate $P$ down so that $f_{0}$ lies in the plane $z=z_{1}$ (say, the Tropic of Capricorn). Rotate $P$ so that one of $f_{0}$ 's vertices lies on the $y$-axis. Then the coordinates of that point are $\left(0, \sqrt{1-z_{1}^{2}}, z_{1}\right)$. Since $f_{0}$ is equilateral, we can 
easily find that its other two points are at $\left(\frac{\sqrt{3} y_{1}}{2}, \frac{-y_{1}}{2}, z_{1}\right)$ and $\left(\frac{-\sqrt{3} y_{1}}{2}, \frac{-y_{1}}{2}, z_{1}\right)$. Since these two are symmetric around the $y$-axis, we can use the arc between these to find the lowest point of $f_{0}$ on the sphere. The midpoint of the spherical arc is the projection of the midpoint of the Euclidean line between these two points, given by the average of the two points:

$$
m=\left(0, \frac{-y_{1}}{2}, z_{1}\right)=\left(0, \frac{-\sqrt{1-z_{1}^{2}}}{2}, z_{1}\right)
$$

We need its magnitude to project it onto the sphere:

$$
\|m\|=\sqrt{\frac{-\sqrt{\left(1-z_{1}^{2}\right)^{2}}}{2}+z_{1}^{2}}=\sqrt{\frac{1-z_{1}^{2}}{4}+z_{1}^{2}}=\sqrt{\frac{1}{4}+\frac{3}{4} z_{1}^{2}}=\frac{1}{2} \sqrt{3 z_{1}^{2}+1}
$$

The midpoint $m$ had a $z$-coordinate of $z_{1}$ and so, when projected onto the sphere, it has a $z$-coordinate of $\frac{z_{1}}{\|m\|}$. Thus, the lowest point $z_{2}$ of $f_{0}$ on the sphere would be

$$
z_{2}=\frac{z_{1}}{\|m\|}=\frac{2 z_{1}}{\sqrt{3 z_{1}^{2}+1}}
$$

If we move all points of $D$ not in $f_{0}$ below $z_{2}$, then we can translate $P$ down and guarantee that $f_{0}$ still eclipses $P$ from the origin, and thus maintain a valid sphere drawing throughout. Using the Tropic of Capricorn for $z_{1}$ yields a value for $z_{2}$ that is above the Arctic Circle, so using the two familiar latitudes guarantees valid sphere drawings throughout. To make sure all vertices outside $f_{0}$ are below $z_{2}$, we scale $P$ down around the $z$-axis by some constant factor $s$. This scaling has the effect of moving all the vertices not in $f_{0}$ towards the south pole. We can calculate the scale-factor $s$ necessary to move all nodes below $z_{2}$ as follows.

Let $z_{3}$ be the maximum $z$-coordinate of any node in $D$ not in $f_{0}$. We would like to scale the point $\left(x, y, z_{3}\right)$ to $\left(\frac{x}{s}, \frac{y}{s}, z_{3}\right)$, such that when it is projected back onto the sphere, its $z$-coordinate is below $z_{2}$. To project $\left(\frac{x}{s}, \frac{y}{s}, z_{3}\right)$ onto the sphere we first find its magnitude. Since the original point lies on the sphere, we have $x^{2}+y^{2}=1-z_{3}^{2}$ and the magnitude is given by:

$$
\sqrt{\frac{x^{2}}{s^{2}}+\frac{y^{2}}{s^{2}}+z_{3}^{2}}=\sqrt{\frac{x^{2}+y^{2}}{s^{2}}+z_{3}^{2}}=\sqrt{\frac{1-z_{3}^{2}}{s^{2}}+z_{3}^{2}} .
$$

As our goal is to have the scaled, projected point lie below $z_{2}$, so we need to find a value for $s$ such that: $\frac{z_{3}}{\sqrt{\frac{1-z_{3}^{2}}{s^{2}}+z_{3}^{2}}}<z_{2}$. Solving for $s$ gives us: $s>\sqrt{\frac{1-z_{3}^{2}}{\frac{z_{2}^{2}}{z_{3}^{2}}-z_{3}^{2}}}$.

Using the scaling factor guarantees all points outside $f_{0}$ fall below $f_{0}$ 's arcs on the sphere when projected, and thus $f_{0}$ eclipses $P$ throughout the translation, and we can move $f_{0}$ on the sphere down to the plane $z=z_{1}$ with the translation $(0,0,-z)$. 


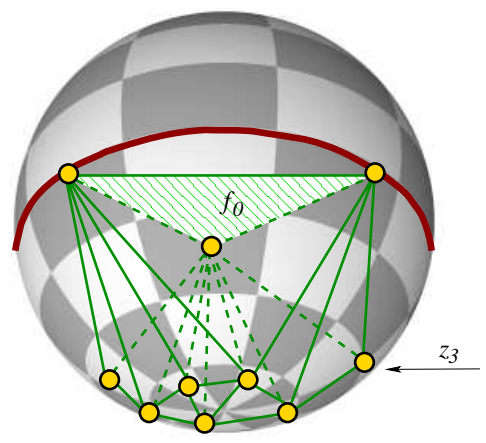

(a)

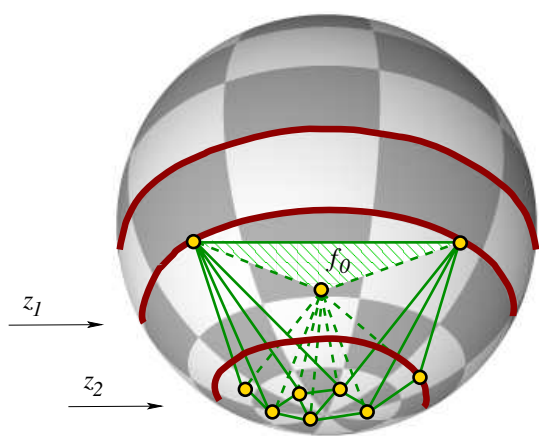

(b)

Fig. 4. The polytope $P$ has a face $f_{0}$ on the equatorial plane. The highest $z$-coordinate of a vertex not on $f_{0}$ is given by $z_{3}$. We would like to translate the polytope straight down so that $f_{0}$ is on the Tropic of Capricorn plane, given by $z=z_{1}$. We ensure that all vertices other than those in $f_{0}$ are below the Antarctic circle, given by plane $z=z_{2}$.

\subsection{The Complete Morph}

We have shown that we can morph a sphere drawing to another sphere drawing that is entirely in one hemisphere. Then, starting with the source drawing $D_{s}$ we can morph it to a drawing $D_{s}^{\prime}$ that is strictly below the equator. We can do the same with the target sphere drawing $D_{t}$ and morph it to a sphere drawing $D_{t}^{\prime}$ that is strictly below the equator. We then obtain the gnomonic projections $D_{s}^{\prime \prime}$ and $D_{t}^{\prime \prime}$ of the two drawings onto the plane tangent to the south pole. We then apply the planar morph algorithm to morph between these two plane drawings. Throughout the planar morph, the sphere drawing is the inverse gnomonic projection of the current state of the plane drawing. Finally, we invert the $D_{t} \rightarrow D_{t}^{\prime}$ morph to arrive at the target drawing.

In order to perform the planar morph, we must ensure that the outer face in $D_{s}^{\prime \prime}$ and $D_{t}^{\prime \prime}$ is the same. We must match the upper faces in $D_{s}^{\prime}$ and $D_{t}^{\prime}$. Theorem 4 allows us to use whichever face we wish, therefore matching is not a problem.

\section{Conclusions and Open Problems}

We have shown that under certain conditions we can morph between spherical drawings such that the morph is continuous and intersection-free. More images and movies are available at http://www.cs.arizona.edu/ mlandis/smorph. Several important open problems remain:

1. Does there exist a continuous and intersection-free morph between any pair of sphere drawings of an underlying 3-connected graph? 
2. In the planar morph stage, what is actually computed is not the trajectories of the vertices, but their locations at any stage in the morph. Is there a morph with trajectories of polynomial complexity?

3. Is there a more direct way to use spherical barycentric coordinates with interpolating between convex representations of graph to obtain a spherical morph, that does not involve reducing the problem to a planar morph?

\section{Acknowledgments}

We thank Alon Efrat, Scott Drysdale and Anna Lubiw, for the stimulating discussions about this problem.

\section{References}

1. P. Alfeld, M. Neamtu, and L. L. Schumaker. Bernstein-Bézier polynomials on circle, spheres, and sphere-like surfaces. Computer Aided Geometric Design Journal, 13:333-349, 1996.

2. B. Aronov, R. Seidel, and D. Souvaine. On compatible triangulations of simple polygons. Computational Geometry: Theory and Applications, 3:27-35, 1993.

3. T. C. Biedl, A. Lubiw, and M. J. Spriggs. Morphing planar graphs while preserving edge directions. In 13th Symposium on Graph Drawing (GD), pages 13-24, 2005.

4. S. S. Cairns. Deformations of plane rectilinear complexes. American Math. Monthly, 51:247-252, 1944.

5. H. Coxeter. Introduction to Geometry. Wiley, 1961.

6. C. Erten, S. G. Kobourov, and C. Pitta. Intersection-free morphing of planar graphs. In 11th Symposium on Graph Drawing, pages 320-331, 2003.

7. M. Floater and C. Gotsman. How to morph tilings injectively. Journal of Computational and Applied Mathematics, 101:117-129, 1999.

8. J. Gomes, L. Darsa, B. Costa, and D. M. Vello. Warping and Morphing of Graphical Objects. Morgan Kaufmann, 1999.

9. C. Gotsman, X. Gu, and A. Sheffer. Fundamentals of spherical parameterization for 3D meshes. In SIGGRAPH '03, pages 358-363, 2003.

10. C. Gotsman and V. Surazhsky. Guaranteed intersection-free polygon morphing. Computers and Graphics, 25(1):67-75, Feb. 2001.

11. J. F. Hughes. Scheduled Fourier volume morphing. Computer Graphics, 26(2):4346, July 1992.

12. A. Lubiw, M. Petrick, and M. Spriggs. Morphing orthogonal planar graph drawings. In 17th Symposium on Discrete Algorithms (SODA), pages 222-230, 2006.

13. T. Samoilov and G. Elber. Self-intersection elimination in metamorphosis of twodimensional curves. The Visual Computer, 14:415-428, 1998.

14. T. W. Sederberg and E. Greenwood. A physically based approach to 2-D shape blending. In SIGGRAPH, pages 25-34, July 1992.

15. V. Surazhsky and C. Gotsman. Controllable morphing of compatible planar triangulations. ACM Transactions on Graphics, 20(4):203-231, Oct. 2001.

16. C. Thomassen. Deformations of plane graphs. J. Combin. Theory Ser. B, 34: 244-257, 1983.

17. W. T. Tutte. How to draw a graph. Proc. London Math. Society, 13(52):743-768, 1963. 